\title{
Effect of Proprioceptive Exercises on Lower Extremity Proprioception in Patients with Diabetic Neuropathy
}

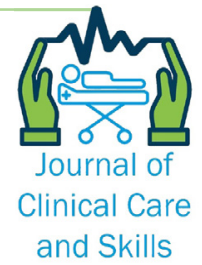

\section{ART ICLE INFO}

\section{Article Type}

Original Research

\section{Authors}

Ghazavi N. ${ }^{1} M S C$,

Sadat SJ.*1 $P h D$,

Roustaei N. ${ }^{2} P h D$

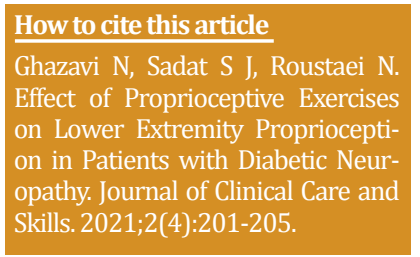

\footnotetext{
${ }^{1}$ Medical Surgical Department, School of Nursing, Yasuj University of Medical Sciences, Yasuj, Iran

${ }^{2}$ Department of Biostatistics and Epidemiology, School of Health, Yasuj University of Medical Sciences, Yasuj, Iran
}

\section{*Correspondence}

Address: School of Nursing, Next to Imam Sajad Hospital, Shahid Ghorbanali Jalil St, Yasuj, Iran. Postal Code: 7591994799

Phone: +98 (74) 33234115

Fax: +98 (74) 33234115

saiedjavadsadat@gmail.com

\section{Article History}

Received: September 5, 2021

Accepted: December 25, 2021

ePublished: January 19, 2022

\section{A B S T R A C T}

Aims Exercise is a unique therapeutic strategy for improving metabolic disorders and risk factors for diabetes and may directly enhance nerve regeneration and function. This study was conducted to determine the effect of proprioception practice on lower extremity proprioception in patients with diabetic neuropathy.

Materials \& Methods This study was an interventional and clinical trial. The population of this study was all patients with diabetic neuropathy who were referred to Abol Abbas and Kourosh clinics in Isfahan in 2020. The sample size in each of the two control groups and proprioceptive exercises group were considered 23 people. Data collection tools included the Michigan Neuropathy Assessment Tool, the Toronto Clinical Rating System, and the goniometer. The proprioceptive exercise was performed for six weeks. Forty-eight hours after the interventions, the goniometer test was performed again. Chi-square, Independent t-test, and Mann-Whitney tests compared the groups using SPSS 21 software.

Findings After the intervention, there was a statistically significant difference between the two groups of proprioception practice with the control group $(\mathrm{p}<0.0001)$. Before and after proprioceptive exercises, there was a statistically significant difference in the proprioceptive exercises group $(\mathrm{p}<0001)$. However, before and after the proprioceptive exercises, there was no statistically significant difference in the control group ( $p>0.05)$.

Conclusion proprioceptive exercises could improve the sense of depth in patients with diabetic neuropathy.

Keywords Glucose Metabolism Disorders; Diabetic Neuropathies; Lower Limb; Proprioception

\section{I T A T I O N L I N K S}

[1] Peripheral neuropathy: evaluation and differential ... [2] Exercise and Manual Therapy for Diabetic Peripheral Neuropathy ... [3] Diabetic neuropathy and diabetic foot. Enfermedades endocrinológicas ... [4] Effects of treatments for symptoms of painful diabetic neuropathy ... [5] A systematic review on the efficacy of vitamin D supplementation on diabetic peripheral ... [6] Physical training and activity in people with diabetic peripheral neuropathy ... [7] Exercise as therapy for diabetic and prediabetic ... [8] The effect of 8 weeks of proprioceptive training on motor coordination in children with autism ... [9] The effect of 6 weeks of training on dynamic balance and proprioceptive ... [10] Exercise training can modify the natural history of diabetic peripheral ... [11] Lifestyle intervention for pre-diabetic ... [12] The effect of eight weeks foot reflexology massage on balance and ankle joint proprioception ... [13] Self care ability of women with diabetes who suffered from peripheral ... [14] A gender-based study of proprioception in the lower ... [15] Intra-examiner reliability of goniometer instrument for all active movements of ... [16] The effect of PNF-stretching and strengthening exercises on improvement of Function ... [17] The effect of two exercise therapy programs (proprioceptive and endurance training) to treat ... [18] Assessment of the impact of proprioceptive exercises on balance ... [19] Sensorimotor and gait training improves proprioception, nerve ... [20] Effects of Tai Chi versus proprioception exercise program on ... [21] The effect of proprioceptive exercises on balance and physical function ... 
Effect of Proprioceptive Exercises on Lower Extremity ...

\section{Introduction}

Peripheral neuropathy is characterized by damage to nerve fibers with small or large diameters of the peripheral nervous system. Damage to smalldiameter nerve fibers leads to changes in motor, sensory, vibrational, and profound sensory functions, and damage to large-diameter nerve fibers causes pain and changes in temperature and automatic function [1]. Diabetic neuropathy is multifactorial and is influenced by genetic, environmental, behavioral, metabolic, neurological, and even vascular factors [2]. The main cause of microvascular and metabolic changes in different types of diabetes is hyperglycemia. These vascular changes damage the primary sensory nerves through neural hypoxia and nutrient deficiency [3]. Careful control of blood sugar and hyperglycemia effectively stops the progression of diabetic neuropathy ${ }^{[4]}$.

Exercise, Transcutaneous Electrical Nerve Stimulation (TENS), and vitamin D supplementation are the most important conservative treatments for reducing pain improving function and balance in diabetic neuropathy [5]. Exercise has also been shown to control blood sugar and reduce cardiovascular risk [2]. Exercise is a unique therapeutic strategy to improve metabolic disorders [6] and improve nerve regeneration and function ${ }^{[7]}$.

Proprioception practice is one of the methods that help to develop and improve sensory and motor function. Proprioception practice aims to increase the nervous system's activity [8]. Balance exercises improve movement control at all levels, an important principle of balance and proprioceptive rehabilitation. Motion control requires reflex responses at the spinal cord level, spontaneous postural and balance responses at the brainstem level, and conscious responses at the cortex level [9]. In longitudinal studies, people with diabetes (without signs and symptoms of diabetic neuropathy) who participated in brisk supervised walking for 4 hours per week had less motor or sensory neuropathy [10]. In another study, diet and exercise modification in people with diabetes resulted in partial skin regeneration, associated with a reduction in neuropathic pain intensity [11].

Previous studies in Iran and other parts of the world have shown the importance of paying attention to the proprioceptive exercises of the lower extremities and the need for appropriate intervention to improve them through non-pharmacological intervention methods. In these studies, the effect of intervention methods on lower extremities proprioception and diabetic neuropathy has been performed in different ways and different groups of patients. However, no study has been found to investigate the effect of profundity training on lower extremity profundity in patients with diabetic neuropathy. Therefore, this study was performed to determine the effect of proprioceptive exercises on lower extremity profundity in patients with diabetic neuropathy.

\section{Materials and Methods}

This study was an intervention and controlled randomized clinical trial that included all patients with diabetic neuropathy who were referred to Abol Abbas and Kourosh clinics in Isfahan in 2020. First, using available sampling method was used. After selecting the eligible participants by block random sampling method, they were assigned to two proprioceptive exercises and the control group. Based on a similar study [12], 21 people in each group were calculated. According to the estimated attrition of $10 \%$ for each group, the sample size was 23 people, and 46 people were recruited. Participants aged 1865 years old, and the exclusion criteria were the presence of disorders other than diabetic neuropathy, such as renal failure or untreated hypothyroidism, using drugs affecting the central nervous system, including anticonvulsants, antiarrhythmics, and immunosuppressive drugs, no history of other chronic diseases, especially advanced heart disease and stroke based on the patient's record, no foot ulcers, no history of dizziness, no orthopedic problems or obstructive gait surgeries, deformity or neurological, rheumatic and musculoskeletal diseases of the lower extremities, no symptoms of central nervous system involvement such as dementia, Parkinson's disease or multiple sclerosis.

Data collection tools included the Michigan Neuropathy Assessment Tool, the Toronto Clinical Rating System, and the 8161EEA goniometer (MSD, Belgium).

The Michigan Neuropathy Assessment Tool has two parts; the first part contains 15 questions about the symptoms of lower limb neuropathy. The second part examines the Achilles reflex of the knee and examines the vibration of the patient's big toe. If the score is at least 2, the patient has neuropathy. The sensitivity and specificity of Semmes Weinstein $10 \mathrm{~g}$ monofilament used in the Michigan Neuropathy Assessment Instrument at -10 points of the sole is $1.64,30.8$, and $64.2-89 \%$, respectively [13].

In the Toronto Clinical Rating System, limb-specific symptoms (up to a maximum of 6 points), reflexes (up to a maximum of 8 points), and sensory disturbances on the thumb (up to a maximum of 5 points) are examined. The total score range of this system is 0 to 19 . The score obtained based on this tool in people with diabetes without neuropathy is $\leq 5$, in people with mild neuropathy is 6 to 8 , in people with moderate neuropathy is 9 to 11 and in cases with severe neuropathy is $\geq 12$. This tool has been used in studies as a reliable clinical tool [13].

The goniometer is used for proprioception measurement (in this study, it was used to calculate 
the proprioception of the ankle joint). The person sits on a chair so that the angle of the trunk with the thigh and the thigh with the knee is at 90 degrees. The evaluator inactively targets the feet of the person at a certain angle called the target angle and asks him to hold the active foot at this angle and memorize it for 5 seconds. These angles are 10 degrees for Dorsiflexion, 20 degrees for Plantar Flexion, 15 degrees for Inversion, and 10 degrees for Eversion. The difference between the angle set by the individual and the angle that the examiner considers for the individual is statistically examined as the error angle [14,15].

Ethical approval was received from the Ethics Committee of Yasouj University of Medical Sciences. After obtaining informed consent from patients to participate in the study, participants were divided into two intervention (proprioceptive exercises) and control groups. In the proprioceptive exercises group, patients were told general information about the exercises, the number of class sessions, and the duration of the sessions. Exercises were performed for six weeks and three sessions per week, each session for 30 minutes and a total of 18 sessions individually for patients to match place and time. The sessions began with warm-up exercises for 5 minutes. The main exercises included: 1 . Maintaining the position: standing on one leg, jumping to the sides and forward-backward; 2- Strength: strengthening the front of the thigh, back of the thigh, squat and sides of the thigh; Plyometric: jumps (one-legged two-legged, 90- and 180-degree rotations) and 4Speed and agility exercises: figure-eight running, running forward-backward and sideways (straight and spiral paths). Exercise intensity was adjusted based on patients' abilities. The number and duration of exercises in the first two sessions were the same and then changed according to patients' ability. Then, 48 hours after the end of the interventions in the proprioceptive exercises group, again, the groups were assessed using a goniometer.

Data were analyzed by SPSS 21 software and inferential tests with a 95\% confidence level. Chisquare test, independent t-test, and Mann-Whitney test were used to compare groups and paired t-test was used to compare the groups before and after the intervention.

\section{Findings}

The mean age in the proprioceptive exercises group was $51.86 \pm 9.77$ years and in the control group was $55.43 \pm 9.09$ years, which were not significantly different ( $\mathrm{p}=0.207$; Table 1).

The distribution of Michigan Neuropathy Assessment Tool scores in the two groups was statistically significant $(\mathrm{p}<0.01)$, but the distribution of Toronto clinical Rating System scores in the two groups were not statistically significant $(\mathrm{p}<0.05$; Table 2$)$.
Ghazavi N. et al.

Table 1) Demographic variables of the study groups $(n=46)$

\begin{tabular}{|c|c|c|c|}
\hline Variable & $\begin{array}{l}\text { Exercises } \\
\text { group }\end{array}$ & $\begin{array}{l}\text { Control } \\
\text { group }\end{array}$ & p-value \\
\hline \multicolumn{4}{|l|}{ Gender } \\
\hline Male & $7(30.4)$ & 5 (21.7) & 0.438 \\
\hline Female & $16(69.6)$ & $18(78.3)$ & \\
\hline \multicolumn{4}{|l|}{ Occupation } \\
\hline Housewife & $15(65.2)$ & 18 (78.3) & 0.247 \\
\hline Manual worker & $5(21.7)$ & $2(8.7)$ & \\
\hline Employee & $2(8.7)$ & 0 & \\
\hline Others & $1(4.3)$ & $3(13.0)$ & \\
\hline \multicolumn{4}{|l|}{ Education } \\
\hline Below diploma & $14(60.9)$ & 21 (91.3) & 0.011 \\
\hline Diploma & $3(13.0)$ & $2(8.7)$ & \\
\hline Above diploma & $6(26.1)$ & 0 & \\
\hline
\end{tabular}

Table 2) Comparison of Michigan Neuropathy Assessment Tool scores and Toronto Clinical Rating System between the study groups.

\begin{tabular}{|c|c|c|c|}
\hline \multirow[t]{2}{*}{ Variable } & Exercises group & Control group & \multirow{2}{*}{$\begin{array}{l}\text { P- } \\
\text { value }\end{array}$} \\
\hline & Median $(Q 1, Q 3)$ & Median $(Q 1, Q 3)$ & \\
\hline $\begin{array}{l}\text { Michigan } \\
\text { Neuropathy } \\
\text { Assessment Tool }\end{array}$ & $(10,12)$ & $(11,14)$ & 0.005 \\
\hline $\begin{array}{l}\text { Toronto Clinical } \\
\text { Rating System }\end{array}$ & $(8,15)$ & $(6,13)$ & 0.147 \\
\hline
\end{tabular}

Q1: First quarter; Q3: Third quarter

According to the information presented in Table 3, the mean score of proprioception before the intervention in the experimental group was higher than the control group. Still, after the intervention, in the experimental group, this value reached 4.17 less than the control group. There was a statistically significant difference before and after proprioceptive exercises in the proprioceptive exercises group $(\mathrm{p}<0.05)$. However, there was no statistically significant difference in the control group $(\mathrm{p}<0.05$; Table 2).

Table 3) Comparison of mean between intra-group and intragroup scores in study groups before and after the intervention

\begin{tabular}{llll}
\hline Intervention & $\begin{array}{l}\text { Exercises } \\
\text { group }\end{array}$ & $\begin{array}{l}\text { Control } \\
\text { group }\end{array}$ & p-value \\
\hline Before & $14.31 \pm 3.11$ & $13.32 \pm 3.26$ & 0.299 \\
After & $9.46 \pm 2.97$ & $13.63 \pm 3.2$ & $<0.0001$ \\
p-value & $<0.0001$ & 0.152 & - \\
\hline
\end{tabular}

\section{Discussion}

This study aimed to determine the effect of profundity training on lower extremity proprioception in patients with diabetic neuropathy. Since researchers have not yet conducted a study examining the effect of profundity training on lower extremity proprioception in patients with diabetic neuropathy, this study seems to be new in this field. The presented findings confirmed the similarity of participants (intervention and control groups) at the beginning of the study in terms of lower extremity proprioception. Therefore, the changes observed after the intervention in the lower extremities proprioception as a dependent variable were due to the effect of proprioceptive exercises as an independent variable of the study. 
Effect of Proprioceptive Exercises on Lower Extremity ..

The present study results showed that 48 hours after the end of the interventions in the proprioceptive exercises group, there was a statistically significant improvement in the level of the lower extremities proprioception. Consistent with this finding, Ghiasi et al. examined the effect of proprioceptive stretching and strength exercises on improving lower limb function in people with tibial stress syndrome. Their results confirmed the effects of strength exercises and proprioceptive-stretching exercises in reducing disability and pain in patients with tibial stress syndrome in the short term [16]. In this regard, Arami et al. showed that ten sessions of endurance training program and deep proprioceptive exercises program in the neck area could improve tolerance, proprioception error, and pain in patients with chronic nonspecific neck pain [17]. Duman et al. showed that proprioceptive exercises have beneficial effects on proprioception. Finally, exercises aimed at eliminating proprioception dysfunction effectively treated osteoarthritis of the knee [18]. The results of this study are consistent with the results of the present study in terms of positive effects on proprioceptive exercises and intervention on the lower limbs. Also, the proprioceptive exercises used, such as walking and running on both sides (spiral paths), were similar in the two studies.

In a similar study, the effect of sensorimotor training and walking for eight weeks (3 days per week) and diabetes education and foot care on proprioception, neural function, and electromyographic activity of lower limb muscles were shown in patients with diabetic peripheral neuropathy. The proprioception was significantly improved in the intervention group's forward, backward, left, and right directions. Balance exercises also stimulate the mechanical receptors in the spindle, tendon limbs, and joint capsule, which are responsible for increasing the sensory inputs of the foot, ankle, and trunk. In addition, walking practice, which included repetitive small movements of the ankle and knee, improved proprioception [19]. In the mentioned study, the instrument used was the Michigan Neuropathy Screening Tool, which was used in the present study to determine proprioception. In both studies, sensorimotor training improved profundity in diabetic peripheral neuropathy. Gradually increasing the difficulty of sensory-motor exercises and walking leads to strengthening the sense of depth because learning is one of the facts that help improve the lower extremities' proprioception [19].

Inconsistent with this study, Liu et al. assessed the effect of tai chi training program with proprioceptive exercises on neuromuscular ankle function in the elderly and showed that none of the interventions had positive effects on neuromuscular function in the ankle. However, the subjects were more interested in and satisfied with tai chi than proprioceptive exercises [20]. Proprioceptive exercises reinforce the information sent and processed by the central nervous system. These exercises combine proprioceptive with other components of balance, such as the vestibular system (inner ear), body shape (cognitive), supportive, body symmetry (trunk), trunk movements, and center of gravity. Although proprioception is an essential element of balance, Proprioceptive exercises are used to improve the integration of kinesthetic and kinesthetic senses in the brain, that is, to increase synaptic connections to understand static and dynamic situations in space [21]. Because sampling was performed during the COVID19 pandemic, the number of diabetic patients referred to medical centers was reduced, and prolonged sampling time. The strength of this study was the interest of the study participants who continued the research despite the pandemic.

Since the client's purposeful relationship with the nurse is one of the main topics of the nursing profession, it is suggested that if the results are obtained in future studies, be used in clinics and treatment centers to improve nervous system function in patients with diabetic neuropathy and also members of the health team consider the implementation of this treatment for patients with diabetic neuropathy. It is also recommended that this study be performed in other diseases that lead to sensory disturbances of the lower extremities proprioception.

\section{Conclusion}

Based on the findings of this study, proprioceptive exercises could improve the proprioception of patients with diabetic neuropathy. Proprioceptive exercises promote movement control at all levels by increasing the function of the nervous and muscular systems, which is an important principle of proprioception rehabilitation.

Acknowledgments: The authors are thankful for the cooperation of Dr. Hossein Marie Ariad, the Yasouj University of Medical Sciences Vice Chancellor for Research, Professor Ardeshir Afrasiabifar, the Yasouj School of Nursing Vice Chancellor for Research and Postgraduate Studies, and also the patients participating in the research.

Ethical Permissions: The ethics committee of Yasouj University of Medical Sciences approved the study (025.1399.-IR.YUMS.REC). The study was also registered at the Iranian Clinical Trial Center with IRCT20200502047260N1.

Conflicts of Interests: This article is taken from the dissertation of a master's degree student in internal medicine and surgery at Yasouj University of Medical Sciences.

Authors' Contribution: Ghazavi N (First Author), Main Researcher/Introduction Writer/Methodologist (35\%); Sadat SJ (Second Author), Main Researcher/Methodologist/Discussion Writer (35\%); Roustaei N (Third Author), Assistant Researcher/Methodology/Statistical Analyst (20\%)

Funding/Support: The research project was supported by the Yasouj University of Medical Sciences. 


\section{References}

1- Castelli G, Desai KM, Cantone RE. Peripheral neuropathy: evaluation and differential diagnosis. Am Fam Phys. 2020;102(12):732-9.

2- Hernández-Secorún M, Vidal-Peracho C, MárquezGonzalvo S, Corral-de-Toro J, Müller-Thyssen-Uriarte J, Rodríguez-Sanz J, et al. Exercise and Manual Therapy for Diabetic Peripheral Neuropathy: A Systematic Review. Appl Sci. 2021;11(12):5665.

3- San Miguel FV, Puente DM, Julià JV. Diabetic neuropathy and diabetic foot. Enfermedades endocrinológicas y metabólicas. 2016;12:971-81. [Spanish]

4- Wong MC, Chung JW, Wong TK. Effects of treatments for symptoms of painful diabetic neuropathy: systematic review. BMJ. 2007;335(7610):87.

5- Yammine K, Wehbe R, Assi C. A systematic review on the efficacy of vitamin D supplementation on diabetic peripheral neuropathy. Clin Nutr. 2020;39(10):2970-4.

6- Kluding PM, Bareiss SK, Hastings M, Marcus RL, Sinacore DR, Mueller MJ. Physical training and activity in people with diabetic peripheral neuropathy: paradigm shift. Phys Ther. 2017;97(1):31-43.

7- Singleton JR, Smith AG, Marcus RL. Exercise as therapy for diabetic and prediabetic neuropathy. Curr Diab Rep. 2015;15(12):1-8.

8- Moeini A, Nazemzadegan GH, Rostami R. The effect of 8 weeks of proprioceptive training on motor coordination in children with autism spectrum disorders. J Motor Learn Mov. 2019;10(4):505-17. [Persian]

9- Ashoury H, Raeisi Z, Khodabakhshi M. The effect of 6 weeks of training on dynamic balance and proprioceptive function of lower extremity chronic ankle sprain with basketball players. Journal of Research in Sport Rehabilitation. 2016;4(7):55-63. [Persian]

10- Balducci S, Iacobellis G, Parisi L, Di Biase N, Calandriello E, Leonetti F, et al. Exercise training can modify the natural history of diabetic peripheral neuropathy. J Diabetes Complications. 2006;20(4):216-23.

11- Smith AG, Russell J, Feldman EL, Goldstein J, Peltier A, Smith S, et al. Lifestyle intervention for pre-diabetic neuropathy. Diabetes Care. 2006;29(6):1294-9.

12- Sadeghidehcheshmeh H, Tasoujian E, Omidi Ar. The effect of eight weeks foot reflexology massage on balance and ankle joint proprioception error in elderly men. J Pract StudBiosci Sport. 2016;4(8):123-35. [Persian]

13- Khosravan S, Ahrari Z, Najafi M, Alami A. Self care ability of women with diabetes who suffered from peripheral neuropathy and its related needs based on Orem's self-care model. Med Surg Nurs J. 2015;4(3):44-51. [Persian]

14- Rahmani Z, Sadeghi H. A gender-based study of proprioception in the lower limbs of patients with Multiple Sclerosis. Iranian J Rehabil Res. 2017;3(4):35-42. [Persian] 15- Zamani S, Okhovatian F, Naemi SS, Baghban AA. Intraexaminer reliability of goniometer instrument for all active movements of cervical spine in asymptomatic young women. J Rehab Med. 2016;4(4):57-64.

16- Ghiasi F, Akbari A, Shoroei H. The effect of PNFstretching and strengthening exercises on improvement of Function in subjects with tibial stress syndrome. Pars J Med Sci. 2010;8(1):19-27. [Persian]

17- Arami J, Rezasoltani A, Khalkhali Zm, Rahnama L. The effect of two exercise therapy programs (proprioceptive and endurance training) to treat patients with chronic nonspecific neck pain. J Babol Univ Med ScI. 2012;14:77-84. [Persian]

18- Duman I, Taskaynatan MA, Mohur H, Tan AK. Assessment of the impact of proprioceptive exercises on balance and proprioception in patients with advanced knee osteoarthritis. Rheumatol Int. 2012;32(12):3793-8.

19- Ahmad I, Verma S, Noohu MM, Shareef MY, Hussain ME. Sensorimotor and gait training improves proprioception, nerve function, and muscular activation in patients with diabetic peripheral neuropathy: a randomized control trial. J Musculoskelet Neuronal Interact. 2020;20(2):234-48.

20 - Liu J, Wang XQ, Zheng JJ, Pan YJ, Hua YH, Zhao SM, et al. Effects of Tai Chi versus proprioception exercise program on neuromuscular function of the ankle in elderly people: a randomized controlled trial. Evid Based Complement Alternat Med. 2012;2012:265486.

21- Espejo-Antúnez L, Pérez-Mármol JM, de los Ángeles Cardero-Durán M, Toledo-Marhuenda JV, Albornoz-Cabello $M$. The effect of proprioceptive exercises on balance and physical function in institutionalized older adults: a randomized controlled trial. Arch Phys Med Rehabil. 2020;101(10):1780-8. 